\title{
Nested prospectivity in perception: Perceived maximum reaching height reflects anticipated changes in reaching ability
}

\author{
JEFFREY B. Wagman ANd Lydia L. Morgan \\ Illinois State University, Normal, Illinois
}

\begin{abstract}
Perception of possibilities for behavior is a necessarily prospective (i.e., forward-looking) act. Such prospectivity is highlighted by the fact that, in general, behaviors are nested within behaviors over a number of spatial and temporal scales. Participants reported their maximum vertical reaching height when they expected to walk across the room and (1) reach for an object while standing on the floor, (2) step up on a step stool and then reach for the object, and (3) pick up a plastic rod and use it to reach for the object. The results show that perception of maximum reaching height was action scaled both when participants expected to perform a nested behavior that would change their action capabilities and when they expected to perform a nested behavior that would not do so. Moreover, the results suggest that nested behaviors that change reaching ability in functionally equivalent ways may bring about functionally equivalent changes in perception of maximum reaching height.
\end{abstract}

In order to successfully perform an intended goal behavior (e.g., retrieving an object from a shelf), perceiveractors must be able to perceive whether that behavior is possible, and (if so) how to control their movements so that this possibility is realized. Such possibilities for behavior are known as affordances and are determined by the fit between a perceiver-actor's action capabilities and behaviorrelevant properties of the environment (Chemero, 2003; J. J. Gibson, 1979; Turvey, 1992). A number of studies have shown that perception of affordances reflects this fit (i.e., that perception of affordances is action scaled). For example, perception of whether an aperture affords walking through is relative to the perceiver-actor's shoulder width (e.g., Warren \& Whang, 1987), perception of whether an aperture affords reaching through is relative to hand size (Ishak, Adolph, \& Lin, 2008), and perception of whether an object affords stepping onto (or over) is relative to leg length (e.g., Warren, 1984).

Importantly, a number of studies have also shown that perception of affordances continues to reflect this fit (i.e., continues to be action scaled) even when action capabilities of the perceiver-actor have changed over short or long time scales. For example, perception of whether an inclined surface affords standing on reflects how a heavy backpack changes the perceiver-actor's center of mass (Malek \& Wagman, 2008; Regia-Corte \& Wagman, 2008), perception of whether a rock climbing hold affords grasping reflects the changing level of fatigue during climbing (Pijpers, Oudejans, \& Bakker, 2007), and perception of whether an aperture affords passing through reflects how an object attached to the body changes the width of the body (Higuchi, Takada, Matsuura, \& Imanaka, 2004; Wagman \& Taylor, 2005).

\section{Nestedness of Behavior and Affordances}

Given that affordances are possibilities for behavior (i.e., potential or future relationships between action capabilities and environmental properties), perception of affordances is necessarily a prospective (i.e., forward-looking) act (Turvey, 1992; Wagman, 2008; cf. E. J. Gibson, 1994). Such prospectivity is highlighted by the fact that, in general, behaviors (and thus affordances) are nested over a number of different spatial and temporal scales (J. J. Gibson, 1979; Reed, 1996; Stoffregen, 2003; Wagman \& Miller, 2003). One particularly useful taxonomy of the nestedness of affordances is as a means-ends hierarchy (Vicente \& Rasmussen, 1990). This hierarchy consists of three (nested) levels, each represented by a question of relevance to the performance of any goal-directed behavior-why, what, and how. The why level represents an overarching goal to be achieved by performing a given behavior, but it does not represent specific behaviors. The what level represents specific behaviors that would achieve the goal represented at the why level, but it does not represent the means by which to perform such behaviors. In other words, the what level represents possible ends but does not specify possible means. The how level represents the various means available for performing the behaviors represented at the what level. Although nesting is present among affordances at all levels of the hierarchy (Vicente \& Rasmussen, 1990; cf. Wagman \& Malek, 2009), the focus in the research reported here is on the nesting of affordances in the what 
and how levels of this hierarchy. That is, the focus is on the nesting between (intended) behaviors and the means by which those behaviors could be performed.

Several features of a means-ends hierarchy of affordances bear on an empirical investigation of (perception of) nested affordances. First, the relationship between action capabilities and behavior-relevant environmental properties determines what means of performing a given behavior are available to a given perceiver-actor. Consider a person who intends to reach for an object on a shelf. Given a particular relationship between reaching ability and object height, the person may be able to reach the object from his or her current location in space merely by extending his or her arm. Given a different relationship, he or she may not be able to do so (see Carello, Grosofsky, Reichel, Solomon, \& Turvey, 1989; Mark et al., 1997).

Second, the levels that define the hierarchy are relative. That is, in cases where a particular means for performing a behavior is unavailable (or underspecified), the levels of the hierarchy "slide" in such a way that the means become represented as the behavior to be performed at the what level (Vicente \& Rasmussen, 1990). For example, a person who is unable to reach an object by extending his or her arm from his or her current location in space may be able to do so from a different location in space (e.g., a location that is closer to the object) (see Mark et al., 1997). That is, the person may be able to perform the behavior by a given means only after changing his or her spatiotemporal relationship to the object (but while leaving his or her action capabilities unchanged). Under such circumstances, perceiving whether the object is reachable requires perceiving whether the height of the object exceeds his or her current maximum vertical reaching height. That is, perception must reflect the (future) fit between the perceiveractor's current action capabilities and behavior-relevant environmental properties.

Third, there is a many-to-many mapping among affordances at the different levels of the hierarchy (Vicente \& Rasmussen, 1990). Some means of performing behaviors satisfy multiple ends, and some ends can be satisfied by multiple means. Consider a person who intends to reach for an object on a tall shelf. In this case, the relationship between reaching ability and object location may be such that he or she is able to reach the object only after performing a behavior that increases his or her maximum vertical reaching ability (with or without changing the spatiotemporal relationship to the object). One option is to step on an elevated surface (such as a step stool) and then reach for the object. Another option is to grasp an elongated object (such as a stick) and then use it to reach for the object. Under such circumstances, perceiving whether the object is reachable requires perceiving whether the height of the object exceeds one's future maximum vertical reaching height. That is, perception must reflect the (future) fit between the perceiver-actor's future action capabilities and behavior-relevant environmental properties.

Fourth, different means may satisfy the same end in functionally equivalent ways. Despite a number of differences between two nested behaviors (e.g., stepping up on a step stool and picking up an elongated object), such behaviors may, under certain circumstances, bring about functionally equivalent changes in action capabilities (e.g., maximum reaching height).

Given these features of a means-ends hierarchy of affordances, several hypotheses about perception of nested affordances emerge. First, perception of nested affordances should be action scaled. In particular, perception of affordances for a given behavior should be relative to the action capabilities of the perceiver-actor both when the means of performing a given behavior would bring about (future) changes in those action capabilities and when it would not. Second, two different but functionally equivalent means of performing a given behavior (i.e., behaviors that would change action capabilities in functionally equivalent ways) should bring about functionally equivalent changes in perception of affordances for that behavior. These hypotheses are the focus of the experiment reported here.

Participants reported their perceived maximum reaching height when they expected to (walk across the room and) (1) reach for an object while standing on the floor (the floor condition) (2) step up on a step stool and then reach for the object (the step stool condition), and (3) pick up a plastic rod and use it to reach for the object (the stick condition). The height of the step stool and the length of the rod were chosen so that they would be likely to increase actual maximum reaching heights in functionally equivalent ways.

In all conditions, participants expected to perform a nested behavior that would change their spatiotemporal relationship with the object prior to reaching (i.e., walk across the room). Thus, the conditions differed in whether subsequent nested behaviors would bring about changes in maximum vertical reaching height. We expected that perception of maximum reaching height would be action scaled (i.e., relative to actual maximum reaching height) in each condition. In the context of the present experiment, action scaling would occur with differences in perceived maximum reaching heights in conditions in which participants differed in their reaching ability but with no such differences in the ratios of perceived-to-actual maximum reaching height across conditions (cf. Warren, 1984; Warren \& Whang, 1987). Accordingly, we expected that (1) perceived maximum reaching heights would occur at taller object heights in the step stool and stick conditions than in the floor conditions (and that there would be no difference between the step stool and stick conditions) and (2) ratios of perceived-to-actual maximum reaching height would not differ across conditions.

\section{METHOD}

\footnotetext{
Participants

Nineteen female undergraduates from Illinois State University participated in this experiment. All participants received extra credit in their psychology courses in exchange for participation. Informed consent was obtained from all participants prior to data collection. In this experiment, participants made judgments about their maximum vertical reaching ability. In order to avoid a ceiling effect (given height constraints provided by the laboratory and the apparatus), it was required that participants be no taller than $168 \mathrm{~cm}(5 \mathrm{ft} 6$ in.). The mean height of the participants was $160.5 \mathrm{~cm}(S D=4.7 \mathrm{~cm})$.
} 


\section{Materials and Apparatus}

Each participant instructed an experimenter to raise or lower a small white cylindrical object ( $82 \mathrm{~g}$ mass, $4 \mathrm{~cm}$ long, $5 \mathrm{~cm}$ in diameter) until she felt that it was at her maximum reaching height. The object was suspended via a pulley system attached to the top of a vertical surface $(250 \mathrm{~cm}$ tall $\times 120 \mathrm{~cm}$ wide $)$. To create a uniform background behind the object, black fabric was draped over the surface. A tape measure affixed to the back of the vertical surface was used to measure the height of the suspended object. A plastic step stool $(23 \mathrm{~cm}$ tall $\times 40 \mathrm{~cm}$ wide $\times 30 \mathrm{~cm}$ deep $)$ was used in the step stool condition. A $33-\mathrm{cm}-$ long section of PVC pipe $(2.5 \mathrm{~cm}$ in diameter) was used in the stick condition. Red tape was wrapped around the first $10 \mathrm{~cm}$ of the PVC pipe. This end was designated as the "handle" of the object. The purpose of the handle was to ensure that the portion of the stick that extended beyond the fist (and functionally extended the reach of the participant) was approximately of the same height as the step stool. After data collection, a tape measure affixed to the wall was used to measure standing height and actual maximum reaching heights. The tape measure was not visible to the participants during the experiment.

\section{Procedure}

Each participant stood $285 \mathrm{~cm}$ from the vertical surface in a designated area $(50 \times 50 \mathrm{~cm})$ that was centered relative to the vertical surface. At the beginning of each trial in a given condition, the object was initially set at either its highest position $(250 \mathrm{~cm}$ from the floor of the laboratory) or its lowest position $(25 \mathrm{~cm}$ from the floor of the laboratory). In the floor condition, the participant instructed the experimenter to raise or lower the object until she felt that it was at the maximum height that she would be able to reach if she were to walk over and stand in a designated area $(40 \mathrm{~cm}$ wide $\times 30 \mathrm{~cm}$ deep) located $15 \mathrm{~cm}$ in front of the vertical surface. In the step stool condition, the step stool was placed in the designated area in front of the vertical surface, and the participant instructed the experimenter to raise or lower the object until she felt that it was at the maximum height she would be able to reach if she were to walk over and step up on the step stool. In the stick condition, the PVC pipe was placed in the center of the designated area (standing upright, with the "handle" up), and the participant instructed the experimenter to raise or lower the object until she felt that it was at the maximum height she would be able to reach if she were to walk over, stand in the designated area, pick up the stick by the handle with her preferred hand, and use it to reach for the object.

In all conditions, maximum reaching height was defined as the maximum height at which the object could be touched with the fingertips of the preferred hand (or distal tip of the stick held in the preferred hand) with the arm and hand (or fist) fully extended above the head, without lifting either foot off of the support surface (i.e., floor or step stool) and without going up on the toes. In all conditions, the participant was able to "fine tune" the height of the object on a given trial until she was satisfied with its height. After each trial, she closed her eyes while the object height was set for the next trial. The experimenter adjusted the height of the object while sitting behind the vertical surface and was not visible to the participant during the experiment.

Each participant completed all three conditions in blocked fashion, and the order of conditions was randomized across participants. In each condition, each participant completed three ascending trials (in which the object height was initially set at its lowest position) and three descending trials (in which the object height was initially set at its highest position). Ascending and descending trials alternated within a given condition, and the order of the sequence (i.e., whether an ascending or a descending trial was presented first in a given condition) was randomized. As a result, there was a total of 18 trials ( 3 conditions $\times 6$ trials per condition) in this experiment.

At no point during the experiment did any participant approach the surface or attempt to perform any of the behaviors (e.g., reaching for the object, stepping onto the step stool, or picking up the PVC pipe). After all trials had been completed, the experimenter measured each participant's standing height and maximum reaching heights while the participant stood on the floor, stood on the step stool, and held the PVC pipe in her preferred hand (while standing on the floor).

\section{RESULTS}

Mean actual and perceived maximum reaching heights in the three reaching conditions were compared in a 2 (reaching height: actual vs. perceived) $\times 3$ (reaching condition: floor vs. stepstool vs. stick) ANOVA. The ANOVA revealed a main effect of reaching height $[F(1,18)=27.98, p<$ $.001]$; actual maximum reaching heights $(M=212.0 \mathrm{~cm}$, $S D=13.8 \mathrm{~cm})$ were greater than perceived maximum reaching heights $(M=191.2 \mathrm{~cm}, S D=19.2 \mathrm{~cm})$. The ANOVA also revealed a main effect of reaching condition $[F(2,36)=34.58, p<.001]$. Pairwise tests with Bonferroni corrections revealed that maximum reaching heights (1) occurred at greater heights in the step stool condition $(M=206.3 \mathrm{~cm}, S D=20.6 \mathrm{~cm})$ than in the floor condition $(M=190.4 \mathrm{~cm}, S D=14.0 \mathrm{~cm})$, and $(2)$ occurred at greater heights in the stick condition $(M=209.3 \mathrm{~cm}, S D=$ $19.1 \mathrm{~cm}$ ) than in the floor condition. As expected, there was no difference between maximum reaching heights in the step stool condition and in the stick condition $(p=.54)$. The interaction between reaching height and reaching condition was not significant $(F<1)$ (see Figure 1).

Perceived maximum reaching heights were divided by the actual maximum reaching height in each condition. These ratio values (with means ranging from 0.89 in the floor condition to 0.93 in the stick condition) were compared in a one-way ANOVA. As expected, this analysis revealed no difference across the three conditions $(F<1$, $p=.45$ ) (see Figure 2).

\section{DISCUSSION}

Previous research has shown that perception of affordances reflects the action capabilities of the perceiver-actor (i.e., perception is action scaled) even when the action capabilities of the perceiver-actor change over short or long time scales. Under such circumstances, perception of affordances

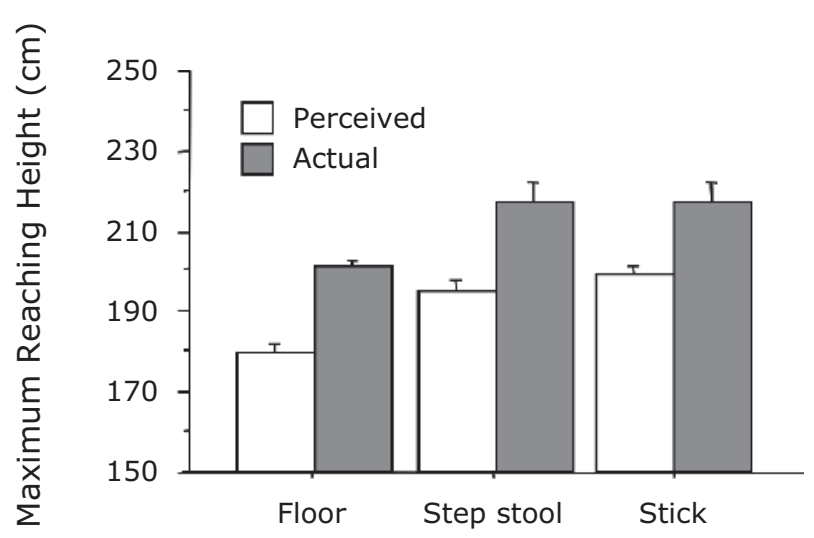

Figure 1. Perceived and actual maximum vertical reaching heights in the floor, step stool, and stick conditions. Error bars represent standard errors. 

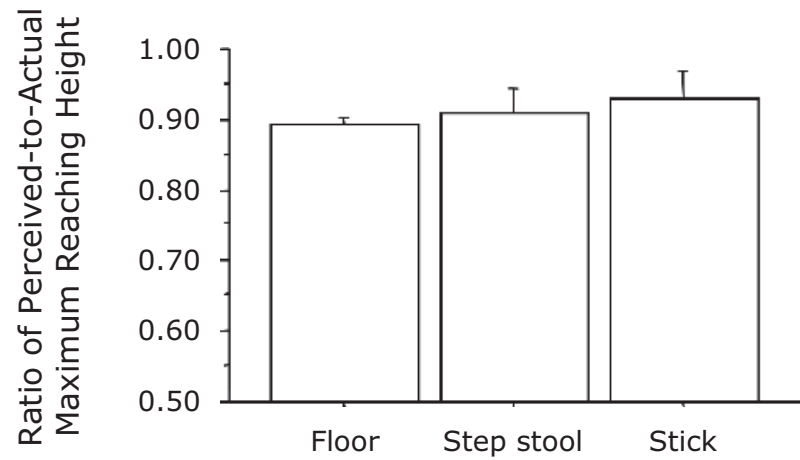

Figure 2. Ratio of perceived-to-actual maximum vertical reaching heights in the floor, step stool, and stick conditions. Error bars represent standard errors.

for a given behavior generally reflects the (new) action capabilities of the perceiver-actor even when the perceiveractor has only relatively limited exposure to such changes (e.g., Ishak et al., 2008; Pijpers et al., 2007; Regia-Corte \& Wagman, 2008). The results of the present experiment build on this body of work by showing that perception of affordances for a given behavior reflects the action capabilities of the perceiver-actor both when the means of performing a given behavior would bring about (future) changes in those action capabilities and when they would not (see also Wagman \& Malek, 2007). That is, perception of affordances for a given behavior reflects the (future) means by which that behavior is to be performed (see Figure 1). Such results are consistent with the means-ends hierarchy of affordances developed by Vicente and Rasmussen (1990).

In addition, previous research has also shown that both action systems and perceptual systems are functionally organized in that they each exhibit anatomical independence. Different anatomical structures can be recruited to perform a given behavior, and a given anatomical structure can be recruited to perform different behaviors (Reed, 1982, 1996). In the same way, different anatomical structures can be recruited to perceive a given environmental property, and a given anatomical structure can be recruited to perceive different environmental properties (see Carello, Fitzpatrick, Domaniewicz, Chan, \& Turvey, 1992; Hajnal, Fonseca, Harrison, Kinsella-Shaw, \& Carello, 2007; Regia-Corte \& Wagman, 2008). The results of the present experiment build on this body of work by suggesting that such functional equivalence may also extend to perception of nested affordances as well. In the context of the present experiment, standing on a step stool and grasping a hand-held object increase the maximum vertical reaching height by different means; but under the right circumstances, they may do so in functionally equivalent ways. The results suggest that the expectation to engage in two different nested behaviors that would change the perceiver-actor's action capabilities in functionally equivalent ways may bring about functionally equivalent changes in perception of affordances. Such results are also consistent with the means-ends hierarchy of affordances developed by Vicente and Rasmussen (1990).
Although perceived maximum reaching height was action scaled in all conditions, there was a small discrepancy between perceived and actual action capabilities (see Figure 1), a finding that is not uncommon in research on perception of affordances (see Mark, 1987; Mark et al., 1997; Regia-Corte \& Wagman, 2008). One possible reason for this is that in most experiments on perception of affordances (including the one reported here), participants are asked to report on the limits of their ability to perform a given behavior, rather than on their preferred means of performing that behavior. Although participants may be able to perform a behavior at the limits of their ability, they may choose another means of performing this behavior if given the opportunity, thus making a report on the limits of their abilities somewhat artificial (see Wagman \& Malek, 2009; Warren, 1984). For example, Mark et al. found that perceived maximum (horizontal) reaching distance in an armonly reaching task differed from the horizontal distance at which participants spontaneously transitioned from an arm-only reach to an arm-plus-torso reach. Whether a similar difference would also occur between perceived maximum vertical reaching height and the height at which there is a spontaneous transition from an unaided vertical reach (i.e., arm only) to an aided vertical reach (i.e., by means of a step stool or hand-held object) may be the topic of future research.

Although the experiment reported here was not designed to explicitly test such proposals, the results are also generally consistent with the proposals that perception and action are embedded and embodied. Respectively, embeddedness and embodiment entail that perception is (1) context dependent and (2) relative to the body and its action capabilities (see Calvo \& Gomilla, 2008; Chemero, 2009). The context in which a perceiver-actor is embedded includes the nesting between (intended) behaviors and the means by which those behaviors could be performed (Vicente \& Rasmussen, 1990). The results of the present experiment show that perception of a given affordance reflects this context. In particular, the results show that perception of maximum reaching height was relative to actual reaching ability (i.e., was action scaled) both when the means of performing this behavior would change participants' reaching ability and when it would not (Figures 1 and 2).

\section{AUTHOR NOTE}

This research was supported by an Illinois State University Faculty Research Award to J.B.W. Correspondence concerning this article should be addressed to J. B. Wagman, Department of Psychology, I1linois State University, Campus Box 4620, Normal, IL 61790-4620 (e-mail: jeffreywagman@ilstu.edu).

Note-Accepted by Cathleen M. Moore's editorial team.

\section{REFERENCES}

Calvo, P., \& Gomilla, T. (EDS.) (2008). Handbook of cognitive science: An embodied approach. San Diego: Academic Press.

Carello, C., Fitzpatrick, P., Domaniewicz, I., Chan, T. C., \& TurVEY, M. T. (1992). Effortful touch with minimal movement. Journal of Experimental Psychology: Human Perception \& Performance, 18, 290-302. doi:10.1037/0096-1523.18.1.290

Carello, C., Grosofsky, A., Reichel, F. D., Solomon, H. F., \& Tur- 
VEY, M. T. (1989). Visually perceiving what is reachable. Ecological Psychology, 1, 27-54. doi:10.1207/s15326969eco0101_3

Chemero, A. (2003). An outline of a theory of affordances. Ecological Psychology, 15, 181-195. doi:10.1207/S15326969ECO1502_5

Chemero, A. (2009). Radical embodied cognitive science. Cambridge, MA: MIT Press.

Gibson, E. J. (1994). Has psychology a future? Psychological Science, 5, 69-76. doi:10.1111/j.1467-9280.1994.tb00633.x

GiBson, J. J. (1979). The ecological approach to visual perception. Boston: Houghton Mifflin.

Hajnal, A., Fonseca, S., Harrison, S., Kinsella-Shaw, J., \& Carello, C. (2007). Comparison of dynamic (effortful) touch by hand and by foot. Journal of Motor Behavior, 39, 82-88. doi:10.3200/ JMBR.39.2.82-88

Higuchi, T., Takada, H., Matsuura, Y., \& ImanaKa, K. (2004). Visual estimation of spatial requirements for locomotion in novice wheelchair users. Journal of Experimental Psychology: Applied, 10, 55-66. doi:10.1037/1076-898X.10.1.55

IsHaK, S., AdOLPH, K. E., \& LiN, G. C. (2008). Perceiving affordances for fitting through apertures. Journal of Experimental Psychology: Human Perception \& Performance, 34, 1501-1514. doi:10.1037/ a0011393

MaleK, E. A., \& Wagman, J. B. (2008). Kinetic potential influences visual and remote haptic perception of affordances. Quarterly Journal of Experimental Psychology, 61, 1813-1826. doi:10.1080/ 17470210701712978

MARK, L. S. (1987). Eyeheight-scaled information about affordances: A study of sitting and stair climbing. Journal of Experimental Psychology: Human Perception \& Performance, 13, 361-370. doi:10.1037/0096-1523.13.3.361

Mark, L. S., Nemeth, K., Gardner, D., Dainoff, M. J., PaAsche, J., DufFy, M., \& GrandT, K. (1997). Postural dynamics and the preferred critical boundary for visually guided reaching. Journal of Experimental Psychology: Human Perception \& Performance, 23, 13651379. doi:10.1037/0096-1523.23.5.1365

PiJPers, J. R., Oudejans, R. R. D., \& BaKker, F. C. (2007). Changes in the perception of action possibilities while climbing to fatigue on a climbing wall. Journal of Sport Sciences, 25, 97-110. doi:10.1080/ 02640410600630894

REED, E. S. (1982). An outline of a theory of action systems. Journal of Motor Behavior, 14, 98-134

REED, E. S. (1996). Encountering the world: Toward an ecological psychology. New York: Oxford University Press.
Regia-Corte, T., \& Wagman, J. B. (2008). Perception of affordances for standing on an inclined surface depends on height of center of mass. Experimental Brain Research, 191, 25-35. doi:10.1007/s00221 $-008-1492-8$

Stoffregen, T. (2003). Affordances are enough: Reply to Chemero et al. (2003). Ecological Psychology, 15, 29-36. doi:10.1207/ S15326969ECO1501_03

Turvey, M. T. (1992). Affordances and prospective control: An outline of the ontology. Ecological Psychology, 4, 173-187. doi:10.1207/ s15326969eco0403 3

Vicente, K. J., \& Rasmussen, J. (1990). The ecology of humanmachine systems II: Mediating "direct perception" in complex work domains. Ecological Psychology, 2, 207-249. doi:10.1207/ s15326969eco0203_2

Wagman, J. B. (2008). Perception-action as reciprocal, continuous, and prospective. Behavioral \& Brain Sciences, 31, 219-220. doi:10.1017/ S0140525X08004032

Wagman, J. B., \& MaleK, E. A. (2007). Perception of whether an object can be carried through an aperture depends on anticipated speed. Experimental Psychology, 54, 54-61. doi:10.1027/1618-3169.54.1.54

WAGMAN, J. B., \& MALEK, E. A. (2009). Geometric, kinetic-kinematic, and intentional constraints influence willingness to pass under a barrier. Experimental Psychology, 56, 409-417. doi:10.1027/1618 $-3169.56 .6 .409$

Wagman, J. B., \& Miller, D. B. (2003). Nested reciprocities: The organism-environment system in perception-action and development. Developmental Psychobiology, 42, 317-334. doi:10.1002/dev .10114

Wagman, J. B., \& Taylor, K. R. (2005). Perceiving affordances for aperture crossing for the person-plus-object system. Ecological Psychology, 17, 105-130. doi:10.1207/s15326969eco1702_3

WARREN, W. H. (1984). Perceiving affordances: Visual guidance of stair climbing. Journal of Experimental Psychology: Human Perception \& Performance, 10, 683-703. doi:10.1037/0096-1523.10.5.683

WARREN, W. H., \& WhANG, S. (1987). Visual guidance of walking through apertures: Body-scaled information for affordances. Journal of Experimental Psychology: Human Perception \& Performance, 13, 371-383. doi:10.1037/0096-1523.13.3.371

(Manuscript received May 2, 2010; revision accepted for publication August 4, 2010.) 